\title{
La diagnosi di infezione da HIV
}

\author{
Alessandra Amendola, Maria Rosaria Capobianchi \\ Laboratorio di Virologia, Istituto Nazionale per le Malattie Infettive "Lazzaro Spallanzani”, Roma
}

\section{Laboratory diagnosis of HIV infection}

\section{INTRODUZIONE}

È stato calcolato che a partire dagli anni 70 , l'infezione da HIV abbia colpito più di 50 milioni di persone al mondo e causato la morte di almeno 16 milioni di individui. Secondo le statistiche WHO e del Joint United Nations Program on HIV/AIDS (UNAIDS), è stato calcolato che alla fine del 1999, circa 33 milioni di individui (adulti e bambini) siano stati contagiati dall'infezione. Nel 1999 sono stati ufficialmente registrati 5,6 milioni di nuovi casi di infezione e le morti correlate con AIDS sono state ben 2,6 milioni. L'infezione da HIV costituisce la seconda causa di mortalità, a livello mondiale, per individui compresi tra 25 e 44 anni. La maggior parte dei nuovi casi di infezione si riscontra nei paesi in via di sviluppo ed interessa prevalentemente individui giovani, con un maggiore aumento soprattutto tra le donne. Le limitate risorse per la prevenzione ed il trattamento dell'infezione e le situazioni di promiscuità, povertà, abuso di droghe, contribuiscono senza dubbio all'ulteriore propagarsi della malattia da HIV.

\section{Sottotipi di HIV}

L'HIV, come tutti i retrovirus, mostra una variabilità genetica molto elevata a causa dei numerosi errori commessi dalla trascrittasi inversa nel processo replicativo. Sono stati identificati due tipi di HIV: HIV-1 e HIV-2. I genomi dell'HIV-1 e dell'HIV-2 presentano una omologia di sequenza intorno al $60 \%$ per i geni che codificano per il core e le proteine polimerasiche, ed una omologia del $30-40 \%$ per i geni che codificano per altre proteine virali.

HIV-1 è diffuso in tutto il mondo e, in base alla sequenza genomica, è stato suddiviso in 3 gruppi ben distinti (tabella 1): gruppo $M$ (major, il più diffuso), il gruppo $O$ (outliers, ceppi altamente divergenti), ed il gruppo $N$ (non-M, non-O). Sulla base delle correlazioni genetiche, all'interno del gruppo $M$ di HIV-1 sono stati identificati più di 10 diversi sottotipi (indicati con lettere alfabetiche, da $\mathrm{A}$ a K) (4). Il sottotipo $\mathrm{B}$ di HIV-1 è il più comune in Europa e negli Stati Uniti; il sottotipo $\mathrm{C}$ è presente in India e in Asia. Esistono inoltre ceppi ricombinanti di HIV-1 il cui genoma si è originato da eventi di ricombinazione fra due o più differenti sottotipi di HIV-1: tali forme sono state denominate CRFs (Circulating Recombinant Forms) (tabella 2). In Tailandia prevale il sottotipo ricombinante $\mathrm{A} / \mathrm{E}$, mentre in Africa sono state rilevate tutte le forme ricombinanti (5).

Il gruppo $O$ di HIV-1 è predominante nelle regioni ovest dell'Africa (Camerun, Gabon, Guinea), tuttavia nuove infezioni da parte di questo gruppo di HIV-1 sono state recentemente registrate anche in Europa e negli USA. A causa dell'eterogeneità dei vari isolati, non sono stati ancora identificati i sottotipi del gruppo $O$.

In analogia con HIV-1, anche per HIV-2 sono stati

Tabella I. II polimorfismo di HIV

\begin{tabular}{|c|c|c|c|c|}
\hline TIPO & GRUPPO & SOTTOTIPO & PREVALENZA & LUOGHI DI MAGGIORE PREVALENZA \\
\hline \multirow[t]{13}{*}{ HIV-I } & $M$ & $\mathrm{~A}$ & Alta & Africa Orientale, Asia \\
\hline & & $\mathrm{B}$ & Alta & Nord e Sud America, Europa, Australia \\
\hline & & C & Alta & India, Africa Meridionale \\
\hline & & $\mathrm{D}$ & Alta & Africa Sub-Sahariana \\
\hline & & $\mathrm{A} / \mathrm{E}_{\mathrm{CM} 240}$ & Alta & Sud-Est Asiatico, Repubblica Centroafricana \\
\hline & & $\mathrm{A} / \mathrm{G}_{\mathrm{IbNG}}$ & Alta & Africa Occidentale e Centro Occidentale \\
\hline & & $\mathrm{F}$ & Moderata & Africa Centro Occidentale, Sud America \\
\hline & & G & Moderata & Africa Occidentale e Centro Occidentale \\
\hline & & $\mathrm{H}$ & Bassa & Africa Centro Occidentale \\
\hline & & $\mathrm{J}$ & Bassa & Africa Centro Occidentale \\
\hline & & K & Bassa & Africa Centro Occidentale \\
\hline & $\bar{N}$ & & Molto Bassa & Camerun \\
\hline & $\mathrm{O}$ & & Molto Bassa & Camerun, Gabon Nigeria, Congo \\
\hline \multirow[t]{5}{*}{ HIV-2 } & & A & Alta & Africa Occidentale \\
\hline & & $B$ & Moderata & Africa Occidentale \\
\hline & & $\mathrm{C}$ & Molto Bassa & Africa Occidentale \\
\hline & & $\mathrm{D}$ & Molto Bassa & Africa Occidentale \\
\hline & & $\mathrm{E}$ & Molto Bassa & Africa Occidentale \\
\hline
\end{tabular}


descritti almeno 5 sottotipi (da A ad E), la cui distribuzione geografica e prevalenza varia considerevolmente. A differenza di HIV-1, l'infezione da HIV-2 è essenzialmente circoscritta all'Africa ed alle poche regioni occidentali in regolare contatto con questi paesi. Le infezioni da HIV-2 del sottotipo A e B sono le più frequenti (tabella 1).

Tabella 2. Ceppi ricombinanti di HIV-I (Circulating Recombinant Forms, CRFs)

\begin{tabular}{|c|c|}
\hline NOMI & SOTTOTIPI \\
\hline CRFOI_AE & $\mathrm{A}, \mathrm{E}$ \\
\hline CRF02_AG & $A, G$ \\
\hline CRF03_AB & $A, B$ \\
\hline CRF04_cpx & $\mathrm{A}, \mathrm{G}, \mathrm{H}, \mathrm{K}, \mathrm{U}$ \\
\hline CRF05_DF & $\mathrm{D}, \mathrm{F}$ \\
\hline CRF06_cpx & $\mathrm{A}, \mathrm{G}, \mathrm{J}, \mathrm{K}$ \\
\hline CRF07_BC & $B^{\prime}, C$ \\
\hline CRF08_BC & $B^{\prime}, C$ \\
\hline CRF09_? & non ancora descritto \\
\hline CRFI0_CD & C,D \\
\hline CRFII_cpx & A,CRFOI_AE, G,J \\
\hline CRFI2_BF & $\mathrm{B}, \overline{\mathrm{F}}$ \\
\hline CRFI3_cpx & A,CRFOI_AE, G,J,U \\
\hline CRFI4_BG & $\mathrm{B}, \mathrm{G}$ \\
\hline CRFI5_0IB & CRFOI_AE,B \\
\hline
\end{tabular}

\section{Parametri sierologici indicatori di infezione da HIV}

La diagnosi di infezione da parte di HIV si basa sull'identificazione sierologica di una avvenuta risposta immunitaria verso il virus, attraverso la ricerca di anticorpi diretti contro le glicoproteine e le proteine strutturali di HIV. La presenza di anticorpi anti-HIV nel siero viene indicata come "sieropositività". Per rilevare la presenza di anticorpi anti-HIV nel siero si utilizzano il saggio ELISA (Enzyme-Linked Immunosorbent Assays), e il test di conferma, più specifico, Western Blot (WB) $(2,6)$. Secondo le linee guida internazionali, la diagnosi di infezione da HIV può essere eseguita solo sulla base di dati sierologici, cioè sull'accertamento della presenza di anticorpi antiHIV, per questo motivo il test ELISA e il test Western Blot sono gli unici saggi diagnostici riconosciuti ufficialmente validi per la diagnosi di infezione da parte di $\operatorname{HIV}(2,6)$.

Nella storia naturale della malattia, tuttavia, gli anticorpi anti-HIV non rappresentano il primo segnale in grado di dare conferma dell'avvenuto contagio. Infatti, il primo parametro rilevabile dopo l'infezione è la presenza di RNA virale nel plasma, seguito dalla presenza di DNA provirale nei linfo-monociti di sangue periferico (PBMC). L'RNA e il DNA di HIV raggiungono una concentrazione massima nella fase acuta, per poi diminuire a livelli bassi o non rilevabili durante la fase asintomatica, cui seguirà un nuovo aumento di pari passo con la progressione della malattia. Un altro parametro precoce indicatore di infezione è la presenza in circolo dell'antigene p24 di HIV (antigenemia). La concentrazione della proteina p24 raggiunge il picco massimo di concentrazione durante la fase acuta dell'infezione, generalmente precedendo la comparsa degli anticorpi di circa 5-10 giorni. Dopo un breve periodo di tempo in cui persiste a livelli misurabili, la p24 viene catturata da anticorpi specifici sottoforma di immunocomplessi e diventa non più rilevabile. Solo il 30-50\% delle persone infette con HIV presenta livelli di antigenemia misurabile nella fase acuta dell'infezione. Nella fase tardiva dell'infezione, la p24 può essere rilevata in conseguenza dell'eccessiva viremia. Gli anticorpi, in genere, iniziano ad apparire 5-6 settimane dopo l'infezione. Mentre gli anticorpi contro le glicoproteine virali persistono per tutta la vita dell'individuo, il titolo degli anticorpi anti-p24 spesso diminuisce, fino a diventare indeterminabile, nella fase tardiva dell'infezione. Il periodo di tempo che intercorre tra l'infezione e la comparsa dei primi marcatori diagnostici, è noto come "periodo finestra" che ovviamente può essere più $\mathrm{o}$ meno lungo, secondo il parametro considerato (6).

La diagnosi di infezione da HIV in fase precoce è estremamente importante in quanto offre maggiori possibilità di successo nel trattamento farmacologico, consente di adottare le norme necessarie per prevenire le infezioni opportunistiche e scoraggiare comportamenti che potrebbero diffondere la malattia da parte di individui interessati. Nonostante la difficoltà nel rilevare la presenza di anticorpi anti-HIV nel periodo finestra (a causa dell'esiguo titolo anticorpale), la determinazione degli anticorpi rappresenta attualmente il metodo più sicuro per la diagnosi di infezione da HIV. Solo in alcuni casi particolari (fase acuta, ipogammaglobulinemia congenita, neonati nati da madri infette da HIV, etc), la diagnosi su base sierologica necessita del riscontro clinico e della rilevazione di altri parametri virologici (antigene p24, rilevazione degli acidi nucleici, isolamento virale, ecc...), la cui valutazione trova applicazione solo nella conferma dell'avvenuta infezione e/o nel monitoraggio della progressione della malattia, e mai nella diagnosi di infezione (6).

Il continuo sviluppo dei metodi diagnostici ha apportato un notevole miglioramento della sensibilità e specificità dei saggi utilizzati per rilevare la presenza di anticorpi anti-HIV. Già con i test di III generazione era stato ottenuto un guadagno diagnostico di circa 20 giorni sui tempi di rilevabilità degli anticorpi. Un ulteriore passo in avanti è stato fatto poi con i test di IV generazione, che 
permettono di evidenziare contemporaneamente gli anticorpi anti-HIV e l'antigene p24, con un guadagno di circa un'altra settimana di tempo nella diagnosi di infezione. Questi test detti "combinati" trovano l'optimum di applicabilità nelle strutture trasfusionali, dove non si effettua la diagnosi di infezione da HIV, ma vengono eseguiti i test di screening per eliminare le donazioni a rischio di trasmettere il virus.

Oltre ai metodi indiretti, esistono tecniche dirette che permettono di dimostrare la presenza del virus nel plasma prima della comparsa di anticorpi specifici, basate sulla ricerca dell'antigene virale p24 o sull'amplificazione o rilevazione degli acidi nucleici virali. I metodi basati sulla ricerca degli acidi nucleici virali rilevano direttamente l'RNA o il DNA di HIV e sono molto più sensibili del test per la rilevazione della proteina virale $\mathrm{p} 24$.

\section{Gli anticorpi anti-HIV}

L'infezione da HIV è un esempio di infezione virale persistente. Virtualmente tutti gli individui infetti producono anticorpi specifici per HIV. Poiché l'infezione ha un carattere persistente e gli anticorpi permangono per tutta la vita, la comparsa di anticorpi diretti contro il virus indica la presenza di infezione, e non testimonia né un'infezione risolta, né tantomeno indica uno stato di protezione dalla stessa.

I saggi per la rilevazione degli anticorpi anti-HIV rappresentano il principale strumento per la diagnosi di infezione in tutti i pazienti, ad eccezione dei neonati nati da madri infette e dei pazienti in fase acuta di infezione, $i$ quali possono non avere ancora sviluppato una specifica risposta anticorpale. Queste due particolari tipologie di pazienti saranno discusse in seguito.

I test per la ricerca di anticorpi anti-HIV si basano su due tipi di saggi immunoenzimatici: ELISA e Western Blot (WB). Il test ELISA viene utilizzato come test di prima battuta (screening) per rilevare la presenza di anticorpi specifici. Attualmente, l'algoritmo diagnostico approvato e seguito per la ricerca degli anticorpi anti-HIV prevede, in prima fase, il test ELISA ripetuto due volte sullo stesso campione (figura I). Se i due test ELISA ripetuti sullo stesso campione danno esito negativo, il campione è considerato negativo per la presenza di anticorpi anti-HIV. Se uno o entrambi i test ELISA producono un risultato positivo, il risultato finale è considerato virtualmente positivo per la presenza anticorpale; in tal caso è assolutamente necessario eseguire il WB per confermare l'esito positivo del test ELISA. La combinazione dei due test ELISA e WB nella valutazione della presenza di anticorpi anti-HIV garantisce una sensibilità e specificità di risultato estremamente elevata con un valore predittivo pari al $100 \%$.

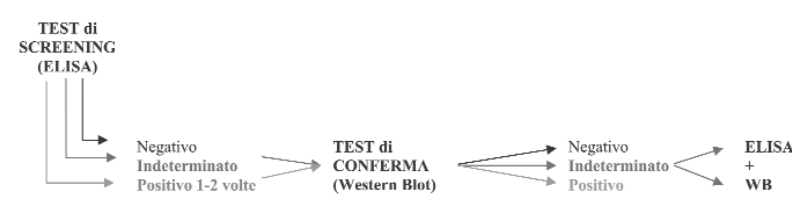

Figura I. Algoritmo per la diagnosi di infezione da HIV in adulti, adolescenti e bambini di età superiore ai 18 mesi.

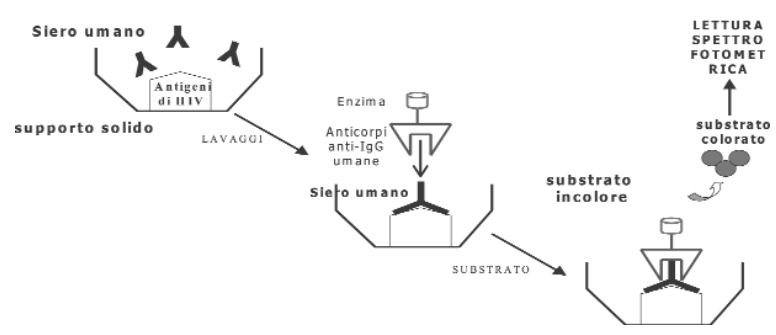

Figura II. Test di screening per la ricerca di anticorpi antiHIV (test ELISA). II test ELISA permette di verificare la presenza di anticorpi anti-HIV nel siero o plasma di un individuo infetto grazie a supporti solidi associati ad antigeni HIV. Se sono presenti, gli anticorpi anti-HIV resteranno ancorati al supporto solido, legati all'antigene riconosciuto. La rilevazione è resa possibile mediante aggiunta di anticorpi secondari, specifici per le immunoglobuline umane, coniugati con un enzima capace di catalizzare la conversione di un substrato appropriato da incolore a colorato. Lo sviluppo di una soluzione colorata al termine del test permette di accertare la presenza di anticorpi anti-HIV. La densità ottica (DO) della soluzione colorata sarà calcolata da uno spettrofotometro.

\section{Il test ELISA}

Il test ELISA (Enzyme Linked Immunosorbent Assay) sfrutta la proprietà di un antigene HIV, legato ad una fase solida (per es. biglie in plastica, piastra per microtitolazione), di catturare anticorpi anti-HIV presenti nel siero o nel plasma di un individuo infetto (figura II). Gli anticorpi catturati possono essere rivelati mediante aggiunta di anticorpi secondari, specifici per le immunoglobuline umane, coniugati con un enzima capace di catalizzare la conversione di un substrato appropriato da incolore a colorato. Lo sviluppo di una soluzione colorata al termine del test permette di accertare la presenza di anticorpi anti-HIV. La densità ottica $(\mathrm{DO})$ della soluzione colorata sarà misurata da uno spettrofotometro e registrata come risultato positivo se la DO è superiore del $10 \%$ rispetto ad un valore soglia (cut-off) calcolato per ciascun test. Il risultato del test ELISA sarà negativo se la DO calcolata sarà inferiore del $10 \%$ rispetto al cut-off, o indeterminato se la DO sarà compresa fra $+/$ - il 10\% del valore di cut-off. 
La preparazione degli antigeni usati nel test è determinante per la specificità del test. Attualmente esiste in commercio una grande varietà di saggi ELISA. Questi differiscono tra loro per l'antigene utilizzato e per la configurazione del test. Alcuni test sono in grado di rilevare contemporaneamente anticorpi specifici per HIV-1 e HIV-2 e quindi sono raccomandati nello screening dei pazienti non selezionati per rischio o nazionalità. La maggior parte dei test utilizza solo antigeni di HIV-1 per catturare gli anticorpi eventualmente presenti nel siero. Questi saggi producono esito positivo nel $60-90 \%$ dei pazienti infettati con HIV-2.

L'elevata purezza degli antigeni virali utilizzati in alcuni test attualmente in uso ha permesso di superare le difficoltà relative alle precedenti versioni, nelle quali l'antigene usato per catturare gli anticorpi era ottenuto da preparazioni parzialmente purificate di HIV, coltivato in cellule di origine umana, e contenenti come contaminanti alcune componenti cellulari. Tali contaminanti erano, di solito, antigeni del complesso di istocompatibilità (HLA) di classe II. Di conseguenza, i sieri provenienti da individui con anticorpi specifici per antigeni HLA (come risultato per esempio di ripetute trasfusioni o in seguito ad esposizione a linfociti fetali durante la gravidanza) risultavano reattivi, ma senza essere infetti. Questo particolare problema è stato minimizzato, se non del tutto eliminato, con i test ELISA di seconda e terza generazione. Nei test di seconda e terza generazione il lisato virale (ottenuto da cellule infettate con HIV e disintegrate con una soluzione contenente un detergente) è stato migliorato nella preparazione, oppure sostituito con antigeni purificati o ricombinanti o con peptici sintetici. Tali sistemi utilizzano porzioni selezionate di antigeni virali, nelle quali sono presenti epitopi immunodominanti e quindi idonei per rilevare una specifica risposta anticorpale. L'uso di preparazioni antigeniche standardizzate e relativamente pure presenta il vantaggio di ottenere risultati più riproducibili. Inoltre, con i test ELISA di terza generazione è oggi possibile svelare anche la presenza di anticorpi specifici per HIV-2. Ciò è stato possibile grazie all'aggiunta, in fase solida, della proteina ricombinante gp36 di HIV-2 come proteina di cattura, consentendo così la ricerca simultanea di anticorpi specifici per i due virus nello stesso campione, senza ulteriore dispendio di tempo o incremento dei costi.

Alcuni test di terza generazione hanno migliorato la loro sensibilità adottando la tecnica del doubleantigen sandwich (DAGS) per scoprire le infezioni acute in fase precoce utilizzando un insieme di antigeni capaci di catturare immunoglobuline $\operatorname{IgG}$ e IgM anti-HIV. Lo stesso gruppo di antigeni, coniugati con un enzima, è utilizzato per rivelare la presenza di anticorpi catturati attraverso una reazione con il substrato appropriato.

I test ELISA attuali sono molto sensibili e specifici nella rilevazione di anticorpi anti-HIV (sensibilità e specificità superiore al $99 \%$ ). Nonostante queste eccellenti caratteristiche, nelle popolazioni a basso rischio di sieropositività una elevata proporzione di test positivi risulta falsamente reattiva. Ciò si verifica in conseguenza del fatto che per un test ELISA esiste comunque una percentuale di reazioni false-positive che, anche se bassa, è inevitabile e, in situazioni di bassa prevalenza, questa assume un valore significativamente elevato (1). Questo fenomeno è descritto dal teorema di Bayes, secondo il quale il valore predittivo positivo di un test diagnostico (e quindi la percentuale di test positivi realmente positivi) è funzione della prevalenza della situazione clinica da diagnosticare nella popolazione. Se la prevalenza è alta, la maggior parte dei test positivi sarà veramente positiva, mentre se la prevalenza è molto bassa, la maggior parte dei test risultati positivi saranno probabilmente falsi positivi, anche se la specificità del test è molto elevata. Per questo motivo le comuni strategie di diagnosi prevedono l'esecuzione di un saggio di conferma (mediante WB) per anticorpi anti-HIV su tutti i campioni risultati ripetutamente reattivi con i saggi di screening. In tabella 3 sono elencate le cause responsabili di risultati ELISA falsi positivi e falsi negativi (1). Trasfusioni multiple, alcune vaccinazioni (ad es. vaccino per il virus influenzale) o manipolazioni improprie del campione sono state associate con test ELISA falsi positivi transienti.

I risultati ELISA falsi-negativi possono essere suddivisi in tre gruppi principali. Il primo gruppo comprende test eseguiti nella fase iniziale dell'infezione o in una fase molto tardiva della malattia. E stato stimato che gli attuali test ELISA producono esito positivo approssimativamente intorno al $22^{\circ}$ giorno dopo l'infezione. Quindi, se il test è eseguito in una fase precoce dell'infezione, il test potrà risultare negativo anche se l'infezione da HIV è presente. Ciò è importante da considerare perché potrebbero essere analizzati individui in fase acuta che mostrano i sintomi clinici simili a quelli della mononucleosi infettiva (febbre, linfoadenopatia e/o rash cutaneo), ma non correlati con la comparsa di anticorpi anti-HIV. È il cosiddetto "periodo-finestra", durante il quale la comparsa di sintomi clinici non è accompagnata da un test ELISA positivo semplicemente per il fatto che il titolo di anticorpi anti-HIV non è abbastanza elevato per essere rilevato dal test. Tuttavia, in questa fase, il titolo del virus nel plasma è gene- 
ralmente molto elevato e $i$ test che rilevano direttamente la presenza del virus, come il dosaggio della p24 o la ricerca dell'RNA virale nel plasma (carica virale), possono risultare utili per evidenziare l'infezione prima della sieroconversione (3). Il test della carica virale è quello che per primo fornisce un risultato positivo in caso di infezione da HIV, e per questo motivo è considerato il test d'elezione nei pazienti con sindrome retrovirale acuta, anche se anche questo necessita comunque di ulteriori conferme. Uno studio condotto di recente ha dimostrato che il test dell'RNA virale plasmatico risulta molto più sensibile anche del test della p24 (il quale pur essendo più specifico del test della carica virale, è meno sensibile). Tuttavia è importante considerare la possibilità di ottenere risultati di viremia (presenza di RNA virale nel sangue) falsamente positivi in individui non infetti da HIV, anche se questi sono facilmente riconoscibili per i bassi livelli di RNA plasmatico che si aggirano intorno a 2000-3000 copie/ml.

I test ELISA possono risultare negativi durante la fase finale della malattia a causa del declino della produzione anticorpale, ma raramente si verifica che il titolo anticorpale scenda al di sotto della soglia di sensibilità del test.

Una seconda categoria di risultati ELISA falsi negativi interessa pazienti che non sviluppano mai una risposta anticorpale HIV-specifica, quali ad esempio coloro che sono affetti da ipogammaglobulinemia congenita, oppure coloro che non sviluppano anticorpi anti-HIV per un lungo periodo di tempo, dopo il quale dimostrano di essere infetti.

Infine, l'incremento della diversità genetica ha portato all'evoluzione di ceppi di HIV-1 che possono risultare non riconosciuti da alcuni test ELISA. Molti isolati di HIV che sono non-reattivi o poco reattivi con test ELISA e WB sono classificati come appartenenti al gruppo $O$. I saggi basati su lisati virali sono molto più efficienti nel rilevare il gruppo $O$ rispetto ai kit che utilizzano antigeni ricombinanti o peptici sintetici, i quali

Tabella 3. Risultati falsi-positivi e falsi-negativi con $i$ test di screening (ELISA)

\begin{tabular}{|c|c|}
\hline \multirow[t]{3}{*}{ Falsi positivi } & Trasfusioni multiple \\
\hline & $\begin{array}{l}\text { Immunizzazione recente contro il virus } \\
\text { influenzale }\end{array}$ \\
\hline & $\begin{array}{l}\text { Manipolazione impropria del campione (per } \\
\text { es. riscaldamento) }\end{array}$ \\
\hline \multirow[t]{5}{*}{ Falsi negativi } & Infezione recente (periodo finestra) \\
\hline & Ipogammaglobulinemia \\
\hline & Infezione allo stadio avanzato (raro) \\
\hline & Sierotipo di HIV-I raro (per es. gruppo O) \\
\hline & Nuovi ceppi o forme ricombinanti (CRFs) \\
\hline
\end{tabular}

sono più ristretti e selettivi verso gli anticorpi rilevati. La reattività immunologia dei ceppi del gruppo $O$ varia da ceppo a ceppo; in uno studio, alcuni ceppi del gruppo $O$ erano reattivi con la maggior parte degli ELISA, mentre altri non erano reattivi con nessuno dei test ELISA provati.

\section{1a. I Test Rapidi}

Oltre ai test ELISA, esistono test rapidi per la determinazione della presenza di anticorpi antiHIV nel siero, i quali sono molto veloci, semplici ed accurati. Questi test hanno trovato impiego soprattutto nei piccoli laboratori diagnostici, nei quali l'esiguo numero di test eseguiti non giustifica la presenza di strumenti necessari per eseguire il test ELISA (lettore, lavatore automatico di piastre), e in particolare nei paesi in via di sviluppo. Uno di questi test utilizza, come fase di cattura solida, una mistura di particelle di lattice coniugate con l'antigene p24 (purificato per affinità) ed il peptide sintetico transmembrana gp41 di HIV. Mediante cartucce per microfiltrazione tutti i reattivi vengono filtrati, concentrati e adsorbiti. Il test si esegue in 10 minuti a temperatura ambiente senza l'apporto di particolari strumenti. Se eseguiti in modo appropriato e interpretati da personale esperto, i test rapidi possiedono una sensibilità ed una specificità simile a quella degli ELISA tradizionali.

\section{1b.Test su urina e saliva}

Oltre che nel sangue, la ricerca di anticorpi antiHIV può essere eseguita anche in altri fluidi corporei, rivelandosi particolarmente utile nei programmi di screening. Gli anticorpi anti-HIV sono presenti a basso titolo nell'urina mentre, nelle secrezioni mucose del cavo orale, sono presenti $\mathrm{ad}$ un titolo più elevato. Esiste un kit che permette di evidenziare la presenza di anticorpi specifici per HIV nelle secrezioni orali, utilizzando un tamponcino assorbente per raccogliere la saliva e una procedura di estrazione. L'analisi si basa su una metodica ELISA la quale, in caso di risultato positivo, dovrà comunque essere confermata mediante WB. L'accuratezza di questo test è eccellente e mostra una sensibilità e specificità (rispettivamente $99.5 \%$ e $99.5 \%$ ) simili a quelle del test eseguito su siero o plasma. Il test sull'urina è leggermente meno sensibile e specifico (rispettivamente $98.7 \%$ e $99.1 \%$ ) rispetto ai test sul siero e sulla saliva. I risultati positivi dovranno comunque essere confermati mediante WB eseguito su siero.

\section{Il Western Blot}

Requisito fondamentale di un saggio di conferma è una specificità elevata, in grado di escludere le false positività di campioni non realmente infetti. 
Secondo le raccomandazioni dell'Organizzazione Mondiale della Sanità, il WB (o immunoblotting) deve essere eseguito come test di conferma, in quanto permette di caratterizzare gli anticorpi diretti contro ciascuna proteina virale (garantendo in tal modo l'assenza di risultati falsi positivi) e dunque di confermare la sieropositività o rilevare le eventuali reazioni non specifiche (6). Il WB differisce dal metodo ELISA nel modo di presentazione dell'antigene al siero sottoposto al test (figura III). Il WB è essenzialmente un ELISA, eseguito su supporto in membrana. Tale procedura prevede la preparazione di strisce di membrana contenenti l'antigene. Una preparazione parzialmente purificata di virus (ottenuta da cellule infettate con HIV e lisate con una soluzione contenente un blando detergente, e purificata mediante sedimentazione in un gradiente) è sottoposta ad elettroforesi su gel di acrilamide per separare le varie proteine virali in base al loro peso molecolare e successivamente trasferita su strisce di membrana di nitrocellulosa. Ciascuna striscia così ottenuta contiene l'intero pannello di antigeni virali. L'esecuzione del WB prevede che il siero da saggiare sia cimentato con una striscia di nitrocellulosa, in modo che gli anticorpi presenti nel campione possano legarsi all'antigene specifico. Gli anticorpi così legati saranno quindi rilevati mediante anticorpi specifici per le immunoglobuline umane coniugati con un enzima in grado di convertire un substrato adatto in un precipitato visibile sulla striscia (figura III).

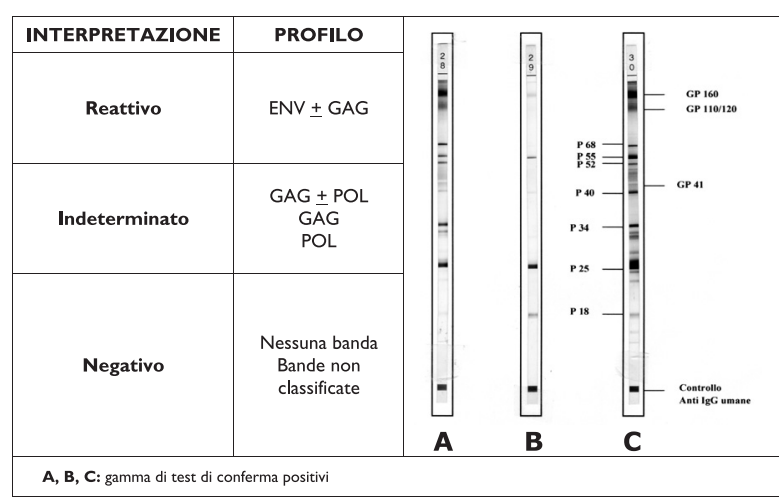

Figura III. Test di conferma per anticorpi anti-HIV:Western Blot. Tale procedura differisce dal test ELISA per il fatto che l'intero pannello di antigeni virali è deposto su strisce di nitrocellulosa, dove ciascun componente è separato dagli altri in base al peso molecolare. Gli anticorpi eventualmente presenti nel campione si legano all'antigene specifico, e possono essere quindi rilevati mediante immunoglobuline specifiche, coniugate con un enzima in grado di convertire un substrato adatto in un precipitato visibile sulla striscia. Le strisce $A B$ e $C$ si riferiscono rispettivamente ad un siero di controllo positivo, fornito dal kit (A); ad un campione di siero positivo per le proteine gpl 20 e p24 (B) e ad un campione di siero contente tutto il pannello di anticorpi specifici per le varie componenti virali (C).
Le strisce del WB si interpretano confrontando la striscia cimentata con il campione da saggiare, con la striscia marcata con il siero di controllo positivo che presenta l'intero corredo anticorpale diretto verso i vari antigeni di HIV. Il test è classificato come reattivo (se sono presenti anticorpi anti-HIV), indeterminato (la presenza di anticorpi anti-HIV non può essere considerata certa, ma non può neanche essere esclusa), o negativo (se gli anticorpi anti-HIV sono assenti).

Secondo i criteri di interpretazione suggeriti dall'Organizzazione Mondiale della Sanità e dal Center of Diseases Control di Atlanta, Georgia, il WB è considerato reattivo quando sono presenti anticorpi specifici per almeno due o più delle seguenti proteine virali, le proteine del core ( $\mathrm{p} 17$, p24, e p55), la polimerasi (p31, p51, p66) e le proteine dell'envelope (gp41, gp120, gp160). L'interpretazione può variare con il kit diagnostico o il metodo. Un WB è sicuramente positivo se è reattivo per gp41 e gp120/gp160, oppure se è reattivo per gp41 o gp120/gp160 e p24. I WB con nessuna banda presente sono considerati negativi. Quando sono presenti una o più bande che non corrispondono a quelle specificate, il blot è considerato indeterminato poiché non risponde al criterio di positività. Errori di interpretazione possono verificarsi in seguito a lettura non corretta delle bande o a contaminazioni del campione negativo da parte di campioni positivi durante la procedura.

\section{2a. Immunoblot indeterminati}

I WB indeterminati sono molto comuni; fino al $15-20 \%$ dei campioni provenienti da individui non-infetti da HIV può produrre risultati di WB indeterminati. Questa è una ragione per cui il WB non è affidabile come test di screening per gli anticorpi anti-HIV. Comunque un risultato indeterminato può essere indicativo di una infezione in fase iniziale $o$ in fase molto avanzata. La presenza di bande corrispondenti ad una o più proteine virali considerate nel criterio di positività, anche se non sono soddisfatti pienamente i criteri di positività, deve essere considerata con sospetto, ed indurre ad ulteriori indagini. Al contrario, $\mathrm{i}$ WB indeterminati che presentano bande che non corrispondono alle proteine essenziali di HIV non indicano quasi mai infezione virale.

Sebbene l'insieme dei test ELISA e WB sia fortemente valido dal punto di vista diagnostico, la scoperta di test ELISA positivi seguita da WB indeterminati può creare uno stato di ansia notevole. Lo stato di infezione di vari individui con WB indeterminato può essere risolto ripetendo il test ELISA e il WB dopo 3-6 mesi per escludere la possibilità che il risultato indeterminato del saggio sia stata conseguenza di un'infezione in 
fase molto iniziale/precoce. Una rapida alternativa è eseguire la PCR per il DNA provirale o un test per la ricerca dell'RNA virale. Entrambi questi test diventano positivi in 1-4 settimane dopo l'infezione virtualmente in tutte le persone infettate recentemente con HIV. Quindi, poiché questi marcatori possono essere ben evidenziati prima della comparsa di anticorpi, un risultato negativo di PCR per HIV DNA o un test negativo per RNA plasmatico esclude un'infezione da HIV come causa di WB indeterminato ed esclude quasi del tutto l'infezione, a meno di una recente esposizione entro le ultime quattro settimane (figura I).

\section{L'antigene virale p24}

L'antigene p24 di HIV (proteina del core virale) può essere rilevato direttamente nel plasma (antigenemia) utilizzando un semplice test ELISA (figura IV). Il tempo medio minimo per la rilevazione della p24 dopo l'infezione è di 16 giorni, 6 giorni prima degli anticorpi anti-HIV. Quindi il test può essere utilizzato per il rilevamento di una infezione acuta da HIV, prima della sieroconversione del paziente, in quanto evidenzia direttamente la presenza del virus in circolo. La concentrazione di p24 nel siero è correlate alla concentrazione del virus infettante nel siero o plasma, quindi il test della p24 può essere indicato nel monitoraggio del decorso clinico della malattia e della risposta del paziente al trattamento farmacologico antivirale. Tuttavia, poiché l'antigene p24 può essere rilevato nel plasma solo in circa il $50 \%$ di individui infetti da HIV, il test è stato ormai quasi completamente soppiantato dalla quantificazione dell'RNA virale nel plasma (metodo molto più sensibile). Altri impieghi del test dell'antigene p24 includono lo screening di donatori di sangue, la diagnosi di infezione da HIV in neonati nati da madri infette da HIV, il monitoraggio della replicazione del virus in-vitro in co-colture di linfociti del sangue periferico. La specificità del test è virtualmente del $100 \%$. Tuttavia, tale livello di specificità richiede la procedura di neutralizzazione per confermare il test iniziale positivo. Nella procedura di neutralizzazione, il campione di siero viene fatto reagire con un anticorpo anti-p24 prima di essere testato. Il test originale è considerato neutralizzabile (veramente positivo per l'antigene p24) se il livello di antigene p24 è ridotto dal pre-trattamento con l'anticorpo.

Alcuni pazienti sviluppano anticorpi anti-p24, di conseguenza il test risulta meno efficiente nel rilevare l'antigene p24, a causa della formazione degli immunocomplessi antigene-anticorpo (figura IV). In tal caso, è necessario un pre-trattamento del siero con acido, base o con il calore per dissociare gli immunocomplessi e rendere l'antigene p24 accessibile alla rilevazione. Nella diagnosi di

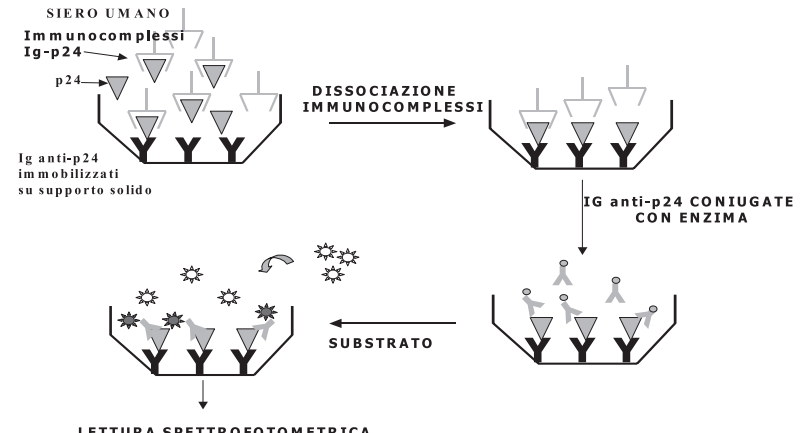

LETTURA SPETTROFOTOMETRICA

Figura IV. II test dell'antigene p24 è un semplice test ELISA che utilizza un supporto solido associato ad anticorpi antip24 per rilevare la presenza dell'antigene virale nel plasma o siero di un individuo infetto. L'abilità del test nel rilevare l'antigene p24 è ridotta se il paziente ha sviluppato anticorpi anti-p24, a causa della formazione degli immunocomplessi antigene-anticorpo. Per questo motivo, il test deve essere preceduto da un procedimento di dissociazione degli immunocomplessi, consistente in un trattamento del siero/plasma con acido, base o con il calore per rendere l'antigene p24 accessibile alla cattura da parte delle immunoglobuline adese alla fase solida.

infezione da HIV nei bambini nati da madri infette, la procedura di dissociazione degli immunocomplessi incrementa la sensibilità del test per l'antigene p24 da circa il 18\% a circa 1' $89,5 \%$. La procedura di dissociazione degli immunocomplessi non è richiesta quando il test si esegue nella fase acuta della malattia, poiché gli anticorpi non sono ancora presenti. Per i test ELISA attuali il periodo finestra è stimato essere di 22 giorni (con un intervallo di confidenza del $95 \%$, da 9 a 34 giorni); il periodo finestra per l'antigene p24 è stimato di 16 giorni.

\section{Isolamento virale}

Con metodi colturali ottimali, il virus può essere isolato dal $97 \%$ dei pazienti che si trovano nella fase asintomatica dell'infezione e dal $100 \%$ di pazienti con AIDS. Attualmente, la coltivazione del virus non viene quasi mai utilizzata a scopo diagnostico, ma rimane una procedura utile per scopi di ricerca, per la sperimentazione di nuovi farmaci antiretrovirali, per risolvere rari casi con risultati conflittuali, per studiare nuove varianti di HIV e il fenotipo del virus.

La procedura-base per coltivare HIV si basa sulla co-coltura di cellule mononucleate di sangue periferico (PBMC) ottenute da un donatore non-infetto con PBMC ottenuti da un individuo infetto. I PBMC isolati dal donatore sano devono essere stimolati con fitoemoagglutinina (PHA) per 1-3 giorni, con l'aggiunta facoltativa dell'interleuchina 2 (IL-2). Successivamente alla stimolazione, si allestisce una co-coltura di queste cellule con PBMC ottenuti da un paziente infetto da HIV, in terreno di coltura RPMI 1640 addizionato con 10- 


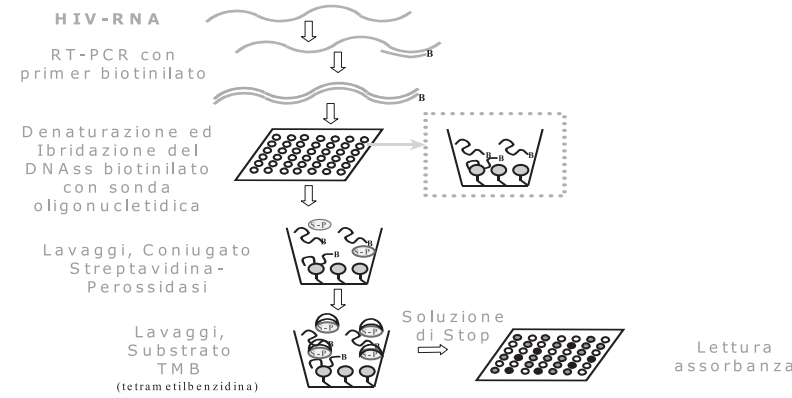

Figura V. Quantificazione della carica virale mediante amplificazione della regione target con metodica RT-PCR. II test rileva e quantifica I'RNA virale presente nel plasma sfruttando la metodica di retrotrascrizione (RT) e reazione polimerasica a catena (PCR). Le particelle virali presenti nel plasma sono disintegrate con guanidina tiocianato e I'RNA virale viene estratto mediante precipitazione con isopropanolo. Mediante trascrizione inversa si procede alla sintesi di copie a DNA complementari all'RNA virale e, in parallelo, ad un RNA competitore interno. I prodotti (cDNA) ottenuti dall'RNA virale e dal competitore interno sono quindi sottoposti alla procedura di PCR. In questa fase si utilizzano primer coniugati con biotina, per ottenere prodotti di amplificazione biotinilati, la cui rivelazione è resa possibile da una reazione enzimatica colorimetrica differenziale, che convertirà un substrato incolore in un substrato colorato. Un analizzatore automatico procederà alla quantificazione delle copie di RNA presenti per millilitro di plasma sulla base di uno standard interno amplificato in parallelo con I'RNA virale presente nel campione (mediante lettura spettrofotometrica della densità ottica). La rilevazione dello standard quantitativo viene eseguita in pozzetti adiacenti.

$20 \%$ di siero fetale bovino, glutammina, IL-2 ed antibiotici. Ad intervalli di sette giorni si aggiungono nuovi PBMC da donatore sano. La replicazione virale può essere rilevata mediante la ricerca dell'antigene virale p24 nel mezzo di coltura ogni 3-4 giorni o, in alternativa, attraverso la dimostrazione dell'attività della trascrittasi inversa. La coltura di HIV può essere inoltre eseguita anche partendo da sangue intero, da plasma o da altri materiali biologici. Per favorire la replicazione virale, e quindi accelerare la positivizzazione della coltura, dai PBMC del donatore sano possono essere eliminati i linfociti CD8, protagonisti di importanti attività anti-retrovirali.

Studi sulla replicazione virale, condotti su linee cellulari continue, hanno dimostrato che alcuni isolati di HIV causano la formazione di cellule giganti multinucleate o sincizi. La presenza di sincizi è stata dimostrata anche in vivo, nei tessuti linfonodali. I ceppi responsabili della formazione di sincizi (SI) sono stati associati ad una rapida progressione della malattia a AIDS, basso numero di linfociti T CD4 e livelli elevati di antigene p24. Studi longitudinali condotti per tre anni hanno dimostrato, nei pazienti infetti da ceppi non-sinciziogeni (NSI), un declino medio della conta dei linfociti T CD4 di 15 cellule/anno, mentre nei pazienti infetti da ceppi SI il declino stimato è di 56 cellule/anno. In un paziente infetto con un ceppo di HIV NSI, la conversione ad un ceppo SI può determinare un rapido declino nella conta delle cellule T CD4 e un rapido aggravarsi delle condizioni cliniche. Dal punto di vista genetico, il fenotipo SI è correlato a cambiamenti nella regione V3 loop del gene env, ma la fisiologia del processo non è stata ancora del tutto chiarita.

\section{PCR per il DNA provirale}

Durante il ciclo di replicazione di HIV, un processo di trascrizione inversa porta alla conversione dell'RNA virale in DNA, chiamato DNA provirale, il quale viene duplicato ed integrato nel genoma della cellula ospite. La metodica convenzionale di amplificazione genica mediante reazione a catena della polimerasi (PCR) può essere utilizzata per rilevare il DNA provirale di HIV nel sangue di tutti gli individui infetti. La principale applicazione della PCR per il DNA provirale è nella diagnosi di infezione da HIV quando la sintesi degli anticorpi è mascherata (come nel caso di infezione neonatale) o non presente, e trova numerose applicazioni anche nel campo della ricerca.

Nella PCR per il DNA di HIV si possono ricercare differenti regioni del genoma. La regione target più utilizzata è $g a g$; tuttavia possono essere utilizzati set di primers specifici per altre regioni del genoma virale ( $p o l$, env). Poiché HIV replica nei PBMC CD4+, tale metodica viene eseguita sul DNA estratto da PBMC purificati o su sangue intero. Una meta-analisi di 96 studi sulla metodica di PCR per rilevare la presenza di DNA provirale di HIV nei PBMC, ha riportato che la sensibilità e la specificità della PCR dipendono dai criteri utilizzati per considerare la reazione di PCR come positiva (per es. uno o più set di primers). Nel complesso, la sensibilità e la specificità delle reazioni di PCR riportate erano, rispettivamente, $97 \%$ e $98.1 \%$. La conclusione degli autori era che i risultati di PCR dovrebbero essere comunque valutati nel contesto del rischio del paziente, della sua storia e delle caratteristiche cliniche. Allo stato attuale, il test PCR resta ancora non standardizzato, e le performance dei test di PCR variano notevolmente fra i laboratori.

Attualmente, la più importante applicazione della PCR per il DNA provirale di HIV è nella diagnosi di infezione da HIV nei bambini nati da madri infette. In due studi eseguiti utilizzando un kit commerciale di PCR per il DNA provirale di HIV (Amplicor HIV-1 Test prodotto da Roche Molecular Systems), la sensibilità del test in bambini esposti al virus era del $50 \%$ durante il primo mese di vita, $96 \%$ da 1 a 6 mesi e $98 \%$ in neonati e bambini al di sopra dei sei mesi di età. La spe- 
cificità era maggiore del $99 \%$. In entrambi gli studi, il test Amplicor HIV-1 era più sensibile della co-coltura nel rilevare l'infezione nei bambini.

Altri vantaggi della PCR per il DNA provirale consistono nel fatto che questa può essere eseguita su un volume relativamente esiguo di sangue, il risultato è disponibile rapidamente, e la performance del test non richiede il livello di contenimento biologico necessario per la coltura di HIV. Le linee guida internazionali attuali suggeriscono che una presuntiva determinazione dell'infezione da HIV nei neonati nati da madre infetta può essere basata sul risultato di due test di PCR, eseguiti uno all'età di un mese o più ed uno all'età di 4 mesi o più (approfondimenti nel paragrafo "Diagnosi di infezione da HIV nei bambini"). Una meta-analisi di 32 studi sulla diagnosi di infezione da HIV nei neonati ha permesso di calcolare una sensibilità media per la PCR del 91.6\% (range 31-100\%) ed una specificità media del $100 \%$ (range 50-100\%). Nel complesso, nei bambini più grandi, la sensibilità e la specificità salgono al $98.2 \%$ rispetto ai neonati (meno di 30 giorni di età) dove è stata valutata intorno al 93.3\%. Con tali parametri un risultato di PCR negativo è associato con una probabilità più alta del $97 \%$ che l'infezione da HIV non sia presente.

\section{Dosaggio della carica virale nel plasma (RNA} di HIV)

La valutazione della carica virale (RNA virale) nel plasma di un paziente rappresenta un parametro fondamentale nella pratica clinica, in quanto fornisce molteplici indicazioni sullo stato di salute del paziente, l'intensità di replicazione virale, la velocità di progressione della malattia e l'efficacia della terapia antiretrovirale.

Attualmente, sono disponibili in commercio varie procedure diagnostiche finalizzate alla misurazione della concentrazione di RNA virale nel plasma di individui infetti. Tali procedure si differenziano tra loro per la tecnologia molecolare impiegata, per la sensibilità, il range dinamico, la regione target di analisi, il volume di campione necessario, la preparazione del campione, ecc. In particolare, le tecnologie molecolari utilizzate per rivelare la presenza del genoma virale si basano o sulla amplificazione della regione target, mediante reazione polimerasica a catena con retrotrascrizione (RT-PCR) ovvero mediante tecnologia NASBA (Nucleic Acid Sequence-Based Amplification), oppure sulla amplificazione del segnale emesso della regione target, sfruttando la tecnologia del branched-chain DNA (bDNA). Una descrizione sommaria dei saggi più in uso è qui riportata.

Per quantificare l'RNA di HIV-1 presente nel plasma di individui infetti, il test "Amplicor HIV
Monitor" di Roche Molecular Systems prevede una prima fase di estrazione ed amplificazione dell'RNA virale mediante RT-PCR ed una seconda fase di rivelazione mediante una tecnica enzimatica (figura V). Dopo disintegrazione delle particelle virali con guanidina tiocianato, l'RNA virale viene estratto mediante precipitazione con isopropanolo. Successivamente si procede alla sintesi copie a DNA complementari all'RNA virale mediante trascrizione inversa, in parallelo ad un RNA competitore interno. I prodotti (cDNA) ottenuti dall'RNA virale presente nel campione in esame e quelli derivati dal competitore interno saranno successivamente sottoposti alla procedura di PCR secondo i metodi convenzionali, utilizzando primer coniugati con biotina, ottenendo così prodotti di amplificazione biotinilati. Questi saranno denaturati, per consentire la cattura di uno dei due filamenti da parte di oligonucleotidi specifici, e rivelati mediante reazione enzimatica colorimetrica differenziale con l'aggiunta di perossidasi di rafano coniugata con straptavidina. Il passaggio finale prevede l'aggiunta di un substrato incolore che sarà convertito in un substrato colorato solo in presenza di perossidasi. Un analizzatore automatico procederà alla quantificazione delle copie di RNA presenti per millilitro di plasma sulla base di uno standard interno amplificato in parallelo con l'RNA virale presente nel campione (mediante lettura spettrofotometrica della densità ottica). Lo standard interno presenta siti di legame dei primer simili a quelli presenti sull'RNA target virale, in modo da poter essere amplificato dagli stessi primer. Invece la sequenza della regione interna dello standard quantitativo differisce dal target in modo da poter essere catturato da differenti sonde di cattura. La rilevazione dello standard quantitativo viene eseguita in pozzetti adiacenti a quelli utilizzati per rilevare l'RNA virale presente nel plasma del paziente (figura V). Questo metodo di quantificazione utilizza come sequenza target di HIV-1 la regione gag. Il range dinamico del metodo standard va da $400 \mathrm{copie} / \mathrm{ml}$ a $750.000 \mathrm{copie} / \mathrm{ml}$. Esiste una versione ultrasensibile del metodo, con limite minimo di sensibilità di 50 copie $/ \mathrm{ml}$ e limite superiore di determinazione della concentrazione di HIVRNA di 75.000 copie/ml.

Il saggio LCx HIV RNA della Abbott Laboratories si basa su una procedura di RT-PCR competitiva (figura VI) per la regione pol. Il risultato della reazione è la produzione di amplicon marcati con due differenti tipi di apteni (uno per lo standard interno ed uno per l'RNA virale) e rivelati mediante metodo MEIA (Microparticle Enzyme Immuno-Assay) da un analizzatore automatico (figura VI). I limiti minimo e massimo di 
sensibilità del metodo sono rispettivamente 50 copie $/ \mathrm{ml}$ e $1.000 .000 \mathrm{copie} / \mathrm{ml}$.

Il test "NucliSens HIV-1 RNA QT" prodotto da Organon Teknika, ora Sanofi bioMérieux, utilizza il metodo NASBA (Nucleic Acid SequenceBased Amplification) per amplificare la regione gag di HIV-1 e quantificare la carica virale plasmatica (figura VII). Dopo aver indotto la lisi delle particelle virali con un tampone adatto, l'acido nucleico virale e tre calibratori interni ad RNA sono sottoposti ad un processo di estrazione mediante un sistema automatico (NucliSens Extractor) che utilizza il metodo di isolamento mediante silice (metodo di Boom). Successivamente gli RNA (sia quello virale presente nel campione, sia i calibratori) sono amplificati secondo il metodo NASBA (il quale coinvolge simultaneamente la trascrittasi inversa del virus della mieloblastosi aviaria, che possiede anche attività RNAasi H, e l'RNA polimerasi T7) per amplificare isotermicamente una sequenza specifica presente sull'acido nucleico con un set di primer specifici per la regione gag, che contengono il promotore per la sintesi dell'RNA da parte dell'RNA-polimerasi T7. A tale scopo, si utilizzano due primers specifici per ottenere l'amplificazione dell'RNA target. Il primer 1 si lega al target ed agisce come innesco per la trascrittasi inversa per la sintesi di una copia a cDNA dell'RNA target.

Il primer 1 inoltre contiene una sequenza che permette l'introduzione di un promotore della RNA polimerasi T7 sul cDNA. La porzione ad RNA dell'ibrido RNA-DNA viene degradato dall'RNAasi H inclusa nella miscela di reazione, lasciando un singolo filamento di DNA copia che include il promotore della T7 RNA polimerasi. Il primer 2 si lega a questo cDNA ed avvia la sintesi del secondo filamento di DNA da parte della trascrittasi inversa. Ne risulta una copia a DNA a doppio filamento, con un attivo promotore T7, utilizzata dalla RNA polimerasi T7 come stampo per la trascrizione di copie multiple di RNA. Queste serviranno come stampo per la sintesi di cDNA e saranno riutilizzate nella serie di reazioni descritte. L'intero processo è isotermico $\left(41^{\circ} \mathrm{C}\right)$. I prodotti di amplificazione (RNA) saranno quantificati in elettrochemiluminescenza attraverso il sistema automatico NucliSens Reader, utilizzando una curva di taratura costruita sui tre standard interni a concentrazione nota e sottoposti alla stessa sequenza di reazioni di amplificazione insieme all'RNA target. Al termine dell'amplificazione i prodotti di reazione sono suddivisi in quattro tubi separati e ciascun componente (RNA target e tre calibratori) è rivelato separatamente mediante specifiche sonde elettrochemilu- minescenti (figura VII). I limiti, minimo e massimo, di sensibilità del metodo sono rispettivamente $80 \mathrm{copie} / \mathrm{ml}$ e $8.000 .000 \mathrm{copie} / \mathrm{ml}$.

Per quantificare la carica virale presente nel plasma di individui infetti da HIV-1 Bayer Diagnostics ha sviluppato la tecnica del branched-chain DNA (bDNA) da vari genotipi di HIV1. Questo metodo (figura VIII) differisce dalla procedura di RT-PCR per il fatto che sfrutta una procedura di amplificazione del segnale emesso da una sonda di DNA ibridata all'RNA virale presente nel campione da testare, anziché procedere all'amplificazione del target. La procedura prevede una fase di ultracentrifigazione, finalizzata alla raccolta di tutte le particelle virali presenti in $1 \mathrm{ml}$ di plasma, una fase di lisi dei virioni con rilascio dell'RNA virale, la cattura dell'RNA virale mediante sonde oligonucleotidiche multiple complementari alle regioni più conservate del gene pol di HIV-1 fissate ad un supporto solido, e la successiva ibridazione con sonde multiple (bDNA). Queste sono successivamente ibridate con oligonucleotidi coniugati con un enzima. In ultima fase l'aggiunta di un substrato chemiluminescente consentirà di accertare la presenza del target e di quantificarlo attraverso l'emissione di un segnale chemiluminescente (la cui intensità sarà proporzionale alla quantità di genoma virale presente), registrato e quantificato da un luminometro. Il numero di copie di RNA virale presente nel campione in analisi è calcolato automaticamente per interpolazione dell'intensità del segnale con la curva di taratura ottenuta utilizzando sei standard interni a concentrazione nota, sottoposti alla stessa procedura in parallelo. Tutte le reazioni, ad eccezione dell'ultima, sono ibridazioni nonenzimatiche puramente quantitative ed offrono il vantaggio di una elevata riproducibilità del metodo (basso coefficiente di variazione) (figura VIII). Il range dinamico del metodo va da 50 copie $/ \mathrm{ml} \mathrm{a}$ 500.000 copie/ml.

Recentemente sono stati approntati saggi basati sull'amplificazione enzimatica delle regioni target del genoma di HIV con rilevazione real time, con metodo TaqMan o con "molecular beacon". Tutti i metodi con rilevazione real time presentano il vantaggio di una significativa riduzione dei tempi di lavoro e di un ampliamento del range dinamico. Alcuni di questi sono stati immessi recentemente sul mercato (es. EasyQ della Sanofi bioMèrieux), altri sono di prossima introduzione (es. test allestito per il sistema a sonde TaqMan della Roche Molecular Systems).

Nonostante le differenze nell'approccio metodologico, le determinazioni della carica virale in campioni infetti dal sottotipo B di HIV-1 ottenute con i saggi in uso risultano significativamente 
correlate e presentano una variabilità interna piuttosto ridotta (approssimativamente $0.12-0.2 \log _{10}$ $\mathrm{copie} / \mathrm{ml}$ su test ripetuti sullo stesso campione).

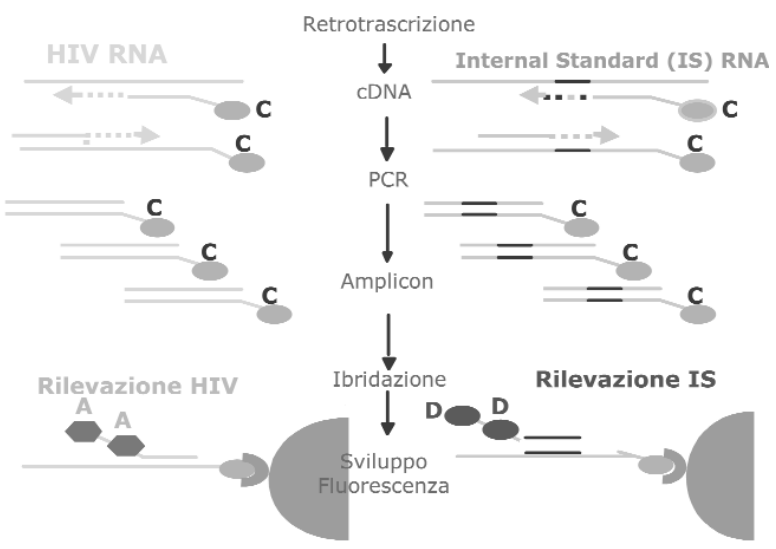

Figura VI. Quantificazione della carica virale mediante amplificazione della regione target con metodica RT-PCR competitiva. II procedimento presenta numerose analogie con quello descritto in Figura IV. II risultato della RT-PCR è la produzione di amplicon marcati con due differenti tipi di apteni (uno per lo standard interno ed uno per l'RNA virale) e rivelati mediante metodo MEIA (Microparticle Enzyme Immuno-Assay) da un analizzatore automatico.

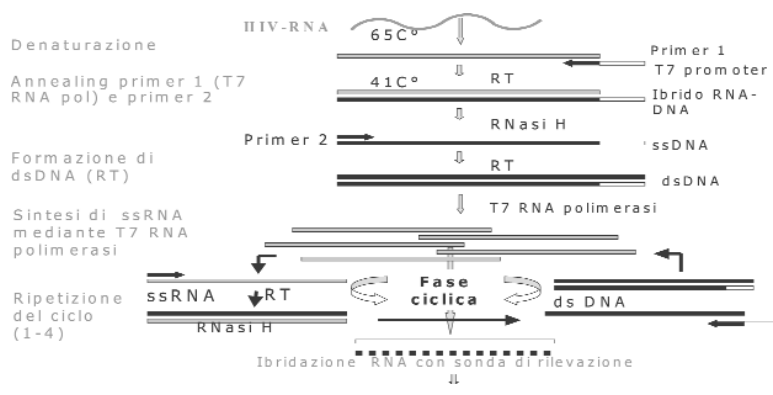

Figura VII. Quantificazione della carica virale mediante amplificazione della regione target con procedura NASBA (Nucleic Acid Sequence-Based Amplification). Dopo l'estrazione dell'acido nucleico virale e di tre calibratori interni ad RNA, gli RNA (sia quello virale presente nel campione, sia i calibratori) sono amplificati secondo il metodo NASBA che utilizza simultaneamente la trascrittasi inversa del virus della mieloblastosi aviaria (che possiede anche attività RNAasi $\mathrm{H}$ ) e l'RNA polimerasi T7 per amplificare isotermicamente una sequenza specifica presente sull'acido nucleico.A tale scopo, si utilizzano due primers specifici per ottenere l'amplificazione dell'RNA target. II primer I agisce da innesco per la trascrittasi inversa per la sintesi di copie a cDNA dell'RNA target. Il primer 2 si lega al singolo filamento di DNA copia ed avvia la sintesi del secondo filamento di DNA da parte della trascrittasi inversa. Ne risulta una copia a DNA a doppio filamento, con un attivo promotore T7, utilizzata dalla RNA polimerasi T7 come stampo per la trascrizione di copie multiple di RNA. Queste serviranno come stampo per la sintesi di cDNA e saranno riutilizzate nella serie di reazioni descritte. L'intero processo è isotermico $\left(41^{\circ} \mathrm{C}\right)$. I prodotti di amplificazione (RNA) saranno quantificati in elettrochemiluminescenza, utilizzando una curva di taratura costruita sui tre standard interni a concentrazione nota e sottoposti alla stessa sequenza di reazioni di amplificazione insieme all'RNA target.

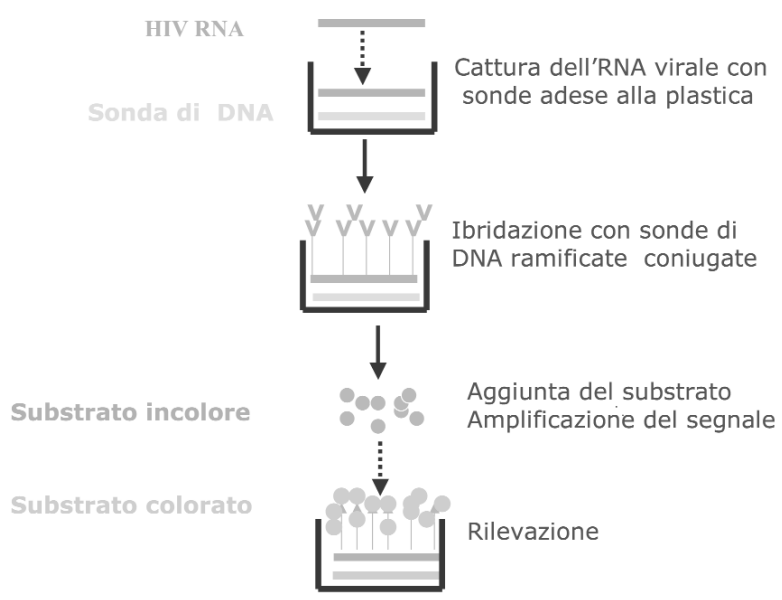

Figura VIII. Quantificazione della carica virale mediante amplificazione del segnale emesso dalla regione target: branched chain DNA (bDNA). Questo metodo permette di quantificare la carica virale presente nel plasma grazie ad una procedura di cattura dell'RNA virale ad un supporto solido mediante sonde oligonucleotidiche multiple complementari, e la successiva ibridazione con vari tipi sonde multiple (bDNA) aggiunte in successione. L'ultima serie di sonde viene associata ad oligonucleotidi coniugati con un enzima che renderà un substrato adatto chemiluminescente. L'emissione del segnale chemiluminescente consentirà di accertare la presenza del target e di quantificarlo (la cui intensità sarà proporzionale alla quantità di genoma virale presente), grazie ad un luminometro.

\section{Diagnosi di infezione da HIV nei bambini}

Secondo le linee guida internazionali, una procedura diagnostica diversa deve essere seguita nel caso di bambini di età inferiore ai 18 mesi, nati da madri infette da HIV. Infatti, il 13-40 \% dei bambini nati da madri sieropositive per HIV contrae l'infezione da HIV. Tale percentuale può essere ridotta approssimativamente del $75 \%$ grazie alla somministrazione della terapia antiretrovirale alla madre ed al bambino. La diagnosi di laboratorio di infezione da HIV in questi bambini è complicata dal trasferimento passivo degli anticorpi materni anti-HIV al bambino. Gli anticorpi di origine materna scompaiono in genere intorno all'età di nove mesi, ma possono persistere fino a 18 mesi (o occasionalmente più a lungo). Per questo motivo, nel periodo in cui sono presenti anticorpi materni, è necessario dimostrare la presenza del virus nel bambino con metodi diretti, quali PCR per il DNA provirale, il dosaggio quantitativo dell'RNA virale nel plasma, il test dell'antigene p24, l'isolamento virale mediante colture cellulari, la ricerca di immunoglobuline IgA anti-HIV nel bambino. Fra questi test, la PCR per il DNA provirale, il dosaggio quantitativo del RNA virale plasmatico e l'isolamento colturale sono i test più sensibili (figura IX).

Numerosi studi riportano che alcuni bambini con- 
traggono l'infezione nell'ultimo periodo della gravidanza o durante la nascita; per questo motivo, durante il primo mese di vita del bambino, $\mathrm{i}$ test che rilevano direttamente la presenza del virus possono dare risultati falsi negativi, poiché la concentrazione del virus nel sangue può essere ancora inferiore ai livelli di sensibilità. Di conseguenza è necessario eseguire una prima analisi entro le prime 24 ore di vita del bambino, in quanto coloro che risultano positivi a questo tempo hanno probabilmente contratto l'infezione inutero e potrebbero avere una progressione più rapida della malattia.

Il metodo virologico preferito per la diagnosi di infezione da HIV nell'infanzia è la PCR per il DNA virale, in quanto permette di evidenziare la presenza del DNA provirale nei linfomonociti di sangue periferico. Anche il saggio che rileva l'RNA virale nel plasma risulta utile per la diagnosi precoce dell'infezione da HIV, tuttavia, i dati sulla sensibilità e la specificità dei test per l'RNA virale sono ancora limitati, rispetto alla PCR per il DNA, e non depongono a favore di una sicura applicazione nella diagnosi precoce. L'isolamento di HIV mediante coltura in vitro mostra una sensibilità simile a quella della PCR per il DNA virale nella diagnosi di infezione da HIV, ma il metodo è più complesso e costoso rispetto alla PCR per il DNA virale e i risultati definitivi possono essere disponibili solo dopo 24 settimane. Possono essere utilizzati anche i test standard per la proteina virale p24 e quelli che prevedono la dissociazione della p24 dagli immunocomplessi, ma la sensibilità di questi test è inferiore rispetto a quella di altri test virologici. L'uso del solo test per l'antigene p24 non è raccomandato per escludere l'infezione o per la diagnosi di infezione nei bambini inferiori ad 1 mese, a causa dell'alta frequenza di test falsi positivi ottenuti in questo periodo di vita del bambino.

Un test virologico positivo è suggestivo di una infezione da HIV, ma richiede la conferma su di un secondo campione. Secondo le linee guida internazionali, il primo test virologico per la diagnosi di infezione da HIV è raccomandato entro le 48 ore dalla nascita su un campione di sangue periferico (figura IX), e grazie ad esso, in questa fase, è possibile identificare quasi il $40 \%$ dei bambini infetti. Successivamente al primo test, altri esami diagnostici dovrebbero essere eseguiti all'età di 1-2 mesi, ed all'età di 3-6 mesi. I bambini con test virologico negativo alla nascita dovrebbero ripeterlo all'età di 14 giorni, dal momento che la sensibilità diagnostica dei test virologici aumenta rapidamente due settimane dopo la nascita, in modo da permettere l'immediata adozione del protocollo di terapia antiretro- virale nel caso di diagnosi di infezione.

I bambini esposti ad HIV e con esito negativo dei test virologici iniziali dovrebbero essere di nuovo esaminati all'età di 1-2 mesi e, nel caso in cui l'esito sia ancora negativo, ancora a 3-6 mesi di età. L'infezione da HIV viene diagnosticata quando due test virologici eseguiti su campioni di sangue prelevati a tempi diversi danno esito positivo. $\mathrm{La}$ presenza del virus può essere ragionevolmente esclusa nei bambini in cui due o più test virologici hanno dato esito negativo, dei quali almeno due siano stati eseguiti all'età 1 mese o più ed almeno un test eseguito all'età di 4 mesi o più. Due o più test negativi per le immunoglobuline $\mathrm{G}(\mathrm{IgG})$ anti-HIV eseguiti all'età di 6 mesi o più, con un intervallo di almeno 1 mese fra i due test, possono essere usati ragionevolmente per escludere un'infezione da HIV fra i bambini che non mostrano alcuna evidenza clinica di infezione. L'infezione può essere definitivamente esclusa se il test per gli anticorpi IgG anti-HIV è negativo (in assenza di ipogammaglobulinemia) all'età di 18 mesi e se il bambino non mostra i sintomi clinici dell'infezione da HIV e i test virologici danno esito negativo (figura IX).

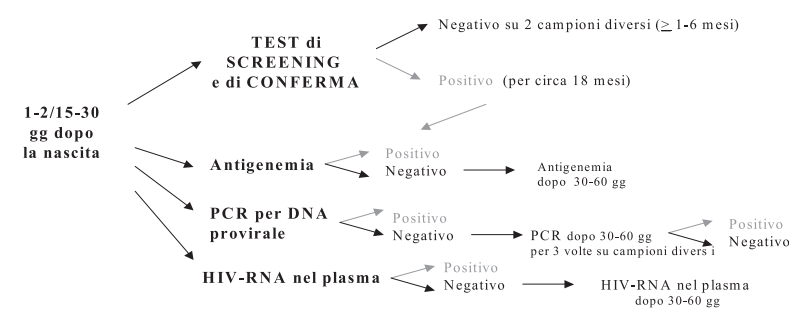

Figura IX. Algoritmo per la diagnosi di infezione da HIV in bambini di età inferiore ai 18 mesi, nati da madri infette da HIV.

\section{Diagnosi di infezione da HIV-2}

Il virus HIV-2 presenta una omologia di sequenza con il genoma di HIV-1 intorno al $60 \%$ in corrispondenza delle regioni gag e pol del genoma, relativamente conservate, mentre per le altre regioni l'omologia di sequenza si aggira intorno al 30-40\%. Come HIV-1, HIV-2 è responsabile di una sindrome da immunodeficienza acquisita, ma questa è caratterizzata da un ritmo di progressione più lento, con un intervallo medio tra il momento dell'infezione e la fase AIDS di 19 anni in assenza di trattamento (mentre per HIV-1 l'intervallo medio è di circa 10 anni). L'infezione da HIV-2 è rara negli Stati Uniti, ma molto più frequente in Europa.

Come per HIV-1, il test fondamentale per la diagnosi di infezione da HIV-2 resta il test ELISA per la ricerca di anticorpi anti-HIV-2. Numerose procedure diagnostiche riescono a rilevare la presenza di entrambi i virus contemporaneamente. I 
test ELISA specifici per HIV-1 risultano reattivi per due terzi dei campioni che contengono anticorpi anti-HIV-2. Esistono inoltre anche test ELISA specifici per HIV-2, i quali vengono utilizzati principalmente nel caso di risultati positivi con $\mathrm{i}$ test ELISA per HIV-1/2 e WB per HIV-1 negativo o indeterminato. Esistono inoltre saggi di WB specifici per HIV-2.

L'algoritmo diagnostico standard per la diagnosi di infezione da HIV-2 richiede, in seguito al test ELISA per HIV-1/2 positivo e al WB per HIV-1 negativo o indeterminato, l'esecuzione di un test ELISA specifico per HIV-2. Se il campione risulta non reattivo con questo test specifico allora viene considerato negativo per la presenza di anticorpi anti-HIV-2. Se invece il saggio ELISA è reattivo su due o tre test, il campione deve essere analizzato con un saggio di WB specifico per HIV-2. Il criterio per considerare il WB positivo per anticorpi anti-HIV-2 stabilisce che devono essere presenti bande che corrispondono alla gp105 (proteina della regione env) e ad una delle seguenti proteine virali: p26, p31, o p55/58/68. Questo algoritmo descrive la procedura più sicura per la diagnosi definitiva di infezione da HIV-2. Una alternativa potrebbe essere l'esecuzione della PCR per il DNA provirale di HIV-2, ma questo test viene eseguito solo in pochi laboratori diagnostici. I sieri contenenti anticorpi anti-HIV-2 analizzati mediante WB per HIV-1 spesso rivelano anticorpi diretti verso le proteine gag e pol (p55, p24, p17, p66, p51, p31) in quanto riconoscono le proteine di HIV-1. Quindi un WB specifico per HIV-1 indeterminato con bande relative ai geni gag e pol, ma non env, suggerisce la presenza di anticorpi anti-HIV-2. La ricerca di anti- corpi anti-HIV-2 è quindi indicata per confermare e diagnosticare l'infezione da parte di HIV-2.

I geni di HIV-2 che codificano per il core presentano un'omologia di sequenza di circa il $60 \%$ con i geni di HIV-1; di conseguenza alcuni test per la ricerca dell'antigene p24 possono essere ugualmente utilizzati per accertare la presenza della proteina $\mathrm{p} 26$, specifica di HIV-2, dal momento che possiedono anticorpi monoclonali multipli. Esistono comunque saggi specifici per la proteina p26 di HIV-2.

Il metodo standard di co-coltura utilizzato per la replicazione di HIV-1 può essere utilizzato anche per isolare HIV-2. La presenza del virus, nel supernatante di crescita, potrà essere confermata mediante test specifico per la proteina p26 di HIV-2 o mediante PCR per il DNA provirale.

\section{BIBLIOGRAFIA}

1. Burke DS, Brundage JF, Redfield RR, et al. Measurement of the false positive rate in a screening program for human immunodeficiency virus infections. N Engl J Med 1988; 319: 961.

2. CDC: US Public Health service Guidelines for testing and counseling blood and Plasma Donors for human immunodeficiency virus type 1 antigen. MMWR 1996; 45 (RR-2): 1.

3. Haseltine WA. Silent HIV infections. N Engl J Med 1989, 320: 1487-9.

4. Hu DJ, Pondero TJ, Rayfield MA, et al. The emerging genetic diversity of HIV. JAMA 1996; 275: 210.

5. Osmanov $\mathrm{S}$, et al. Estimated global distribution and regional spread of HIV-1 genetic subtypes in the year 2000. J Acquir Immune Defic Syndr 2002; 29: 18490.

6. Revised Guidelines for HIV Counseling, Testing and referral. Vol 50, N. RR19; 1. 2001.

\author{
Maria Rosaria Capobianchi \\ Laboratorio di Virologia, \\ Istituto Nazionale per le Malattie Infettive \\ "Lazzaro Spallanzani" \\ Via Portuense 292, 00149, Roma \\ Tel.: 06 55170434; Fax: 065582346 \\ E-mail: capobianchi@inmi.it
}

